\title{
Trefoil Factor 3 (TFF3) mRNA Expression Level in Follicular Thyroid Tumors Using Formalin-Fixed, Paraffin-Embedded (FFPE) Blocks
}

\author{
Saydiganikhodja Ismailov ${ }^{1}$, Murodjon Rashitov ${ }^{1}$, Makio Kobayashi ${ }^{2}$, Noriyuki Shibata ${ }^{2}$, \\ Yoichiro Kato $^{2}$, Yoko Omi ${ }^{2}$, Masatoshi Iihara ${ }^{3}$, Takahiro Okamoto ${ }^{3}$ \\ ${ }^{1}$ Republican Specialized Scientific Practical and Medical Center of Endocrinology, Tashkent, Uzbekistan; ${ }^{2}$ Department of Pathology,
Tokyo Women's Medical University, Tokyo, Japan; ${ }^{3}$ Department of Endocrine Surgery, Tokyo Women's Medical University, Tokyo,
Japan. \\ Email: endocrin@uzsci.net
}

Received November $20^{\text {th }}, 2012$; revised December $20^{\text {th }}, 2012$; accepted January $19^{\text {th }}, 2013$

Copyright (C) 2013 Saydiganikhodja Ismailov et al. This is an open access article distributed under the Creative Commons Attribution License, which permits unrestricted use, distribution, and reproduction in any medium, provided the original work is properly cited.

\begin{abstract}
Background: Differential diagnosis of follicular thyroid carcinoma (FTC) from follicular thyroid adenoma (FTA) is often difficult since presence or absence of capsular/vascular invasion can not be determined by preoperative fine needle aspiration cytology, and may not be judged unanimously on permanent sections even among experienced pathologists. Determination of molecular-genetic factors such as trefoil factor 3 (TFF3) mRNA in the follicular thyroid tumors may be useful aid to improve the accuracy of diagnosis, though it is considered to be unstable and relatively low concentrated genetic substance. Purpose of our study is to investigate expression level of TFF3 mRNA of thyroid follicular tumors using formalin-fixed, paraffin-embedded (FFPE) tissue. Methods: Study population included FFPE sections from 19 FTC cases, 20 FTA cases, 11 adenomatous goiter $(\mathrm{G})$ cases and 12 samples of normal thyroid tissue (N) adjacent to thyroid tumors. RNeasy FFPE kit was used for extraction of total RNA. Purification and concentration values were determined by spectrophotometer. Extracted RNA was used for cDNA synthesis in reverse transcription. Synthesized cDNA subsequently proceeded for relative quantification of TFF3 mRNA by RT-qPCR using TFF3 primers. Glyceroldehyde-3-phosphate dehydrogenase (GAPDH) and hypoxanthin phosphorobosyltransferase1 (HPRT1) were used as control genes. The mean and standard deviation of TFF3 mRNA expression level were analyzed by software Multiplate RQ. Results: Extraction by the FFPE kit yielded high concentration of RNA in all cases. Purification values were 1.8 in average. Concentration values were significantly higher in FTC and FTA relative to G and N tissues, possibly due to high density of thyrocytes in the samples. Relative quantification of TFF3 mRNA expression level showed broad ranges both in FTC and FTA, while the analyses in G and N tissues indicated narrow ranges. Conclusion: FFPE tissues from thyroid follicular tumors can be used for measurement of unstable and low concentrated genetic substances such as TFF3 mRNA. Its diagnostic value yet remains to be determined.
\end{abstract}

Keywords: Follicular Thyroid Tumors; TFF3; RT-qPCR; Formalin-Fixed Paraffin-Embedded Tissue

\section{Introduction}

The histological classification of thyroid epithelial tumors is the current standard to distinguish benign from malignant thyroid tumors and to assign specific tumors into histological subtypes. The World Health Organization (WHO) classification has recently been revised and distinguishes thyroid tumor types [1]. For many of these tumor types, the histological criteria are subjective and thus difficult to implement. Furthermore, reliable biologic prognostic factors are still lacking [2].
Since the introduction of fine needle aspiration biopsy (FNAB) in the early 1970s, diagnosis of the majority of thyroid malignancies, especially that of papillary carcinoma, can be made without much difficulty [3]. However, preoperative diagnosis of follicular carcinoma still remains difficult since capsular or vascular invasion is not possible to determine cytologically. When the tumors can not be diagnosed by cytological examinations, identification of a definite molecular marker is crucial for establishment of a preoperative test to diagnose follicular carcinoma. 
Recently proposed marker genes in thyroid tumors are adrenomedullin-3 (ADM3) [4], cyclin D2 (CCND2), prostate differentiation factor (PLAB), proprotein convertase subtilisin/kexin type 2 (PCSK2) [5], lectin, galactosidebinding, soluble, 3 (LGALS3), trefoil factor 3 (TFF3) [68], beta-1,4-galactosyltransferase (B4GALT), HGD1 [9], thyroglobulin (TG), and v-etserythroblastosis virus E26 oncogene homolog 1 (ETS1) [10].

While other markers failed to show reproducible results in follow-up studies, researches at other institutes revealed the utility of TFF3 mRNA for the diagnosis of thyroid tumors including follicular carcinoma [11-13].

Upregulation of TFF3 is observed in normal thyroid tissues, adenomatous goiters, $80 \%$ of follicular adenomas, and $20 \%$ of minimally invasive follicular carcinomas, while downregulation is observed in anaplastic carcinomas, papillary carcinomas, widely invasive follicular carcinomas, $80 \%$ of minimally invasive follicular carcinomas, and $20 \%$ of follicular adenomas $[7,11,13]$. Thus, TFF3 mRNA is a universal indicator for thyroid tumors derived from thyroid follicular cells.

Thus, TFF3 mRNA is now regarded to be the best marker to distinguish thyroid malignancies including follicular carcinoma. However, there are still several problems to be solved. First of all, TFF3-based diagnosis is not perfectly match with the pathological diagnosis. This discrepancy is the most evident in the diagnosis of minimally invasive follicular carcinoma. This may indicate that the pathological diagnosis of minimally invasive carcinoma does not always reflect the biological features of thyroid tumor cells. So, the reasons for discrepant results obtained between TFF3-based and pathological diagnosis remain unclear.

When a pathologist gives the conclusion that resected thyroid tumor is benign, but recommends follow up, since presence of follicular thyroid carcinoma can not be excluded confidentially, such a follicular adenomas were classified as "suspicious". Our idea is to show that, the useful results may be obtained even using formalin-fixed, paraffin-embedded (FFPE) blocks. Our study is first report that have extracted and examined TTF-3 RNA from the paraffin blocks of follicular thyroid tumors. That is why we choose FFPE tissue for analysis. We did not study cytology specimen. Specimens were microdissected from resected thyroid tissues that had been sent from endocrine surgery department to pathology department. In the available literature we found only few studies concerning RNA studies [14-16] and no study was found on TFF3 expression level in FFPE thyroid tissue.

Thus, the purpose of our study is to investigate recently proposed tumor marker TFF3 mRNA expression level for the differential diagnosis of follicular thyroid adenoma and follicular thyroid carcinoma using FFPE tis- sue.

\section{Materials and Methods}

\subsection{Thyroid Samples}

Diagnosis of follicular thyroid tumors were established according to signs of clinical manifestation, ultrasonography, FNAC, intraoperative inspection, final pathology and classified according to function and histology as previously described $[1,2]$. Expression level of trefoil factor 3 (TFF3) mRNA was studied in formalin-fixed, paraffin-embedded (FFPE) blocks of follicular thyroid tumors. The study population included FFPE sections from 19 follicular thyroid carcinoma (FTC) cases, 20 follicular thyroid adenoma (FTA) cases, 11 adenomatous goiter (G) cases and 12 samples of normal thyroid tissue $(\mathrm{N})$ adjacent to thyroid tumors ( $1-5$ years of archival time). G and $\mathrm{N}$ samples were used as a control group. There are five cases of FTA were classified as a suspicious follicular thyroid adenoma due to the pathological findings and required follow up.

FFPE blocks of thyroid samples were obtained from the Department of Endocrine Surgery, Tokyo Women's Medical University, Japan.

\subsection{RNA Extraction, cDNA Synthesis and RT-qPCR}

Before proceeding to RNA extraction, FFPE blocks of thyroid samples were once more confirmed for their correspondence to assumed thyroid pathology by conventional HE staining using $4 \mu \mathrm{m}$ section. Then, $10 \mu \mathrm{m}$ of FFPE block 4 - 5 sections were collected in to $2 \mathrm{ml}$ tube at sterile conditions. The microtome, all equipment and instruments were cleaned up by $70 \%$ ethanol to avoid RNA degradation and decreasing of RNA yield.

Collected tissue samples were deparaffinized with xylene, homogenized and transferred for RNA extraction. RNA clean up was performed using the RNeasy FFPE Kit (Qiagen Sciences, MD, USA) according to manufactures protocol.

Spectrophotometry of extracted total RNA was carried out to calculate purification and concentration values. Purification value is calculated as ratio A260/A280. Concentration value is calculated according to dilution index as follow: $\mathrm{CV}=\mathrm{A} 260 \times 50 \times 40(\mathrm{ng} / \mu \mathrm{l})(\mathrm{U}-2000 \mathrm{~A}$ spectrophotometer, HITACHI, Tokyo, Japan). In case of low RNA yield the extraction procedure was repeated until enough amount of total RNA is collected.

Total RNA $(1 \mu \mathrm{g})$ was reverse transcribed in a $20 \mu \mathrm{l}$ reaction. The reaction mixture consisted of $4 \mu \mathrm{l} 5 \times$ PrimeScript Buffer, $1 \mu \mathrm{l}$ Enzyme Mix, $1 \mu \mathrm{l}$ oligod NTP,

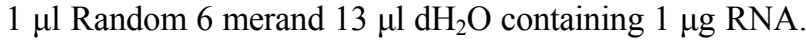
Shortly centrifuged in $10,000 \mathrm{rpm}$ for $10 \mathrm{sec}$. Reverse 
transcription was performed at $37^{\circ} \mathrm{C}$ for $15 \mathrm{~min}$ and at $85^{\circ} \mathrm{C}$ for $1 \mathrm{~min}$. Tube containing synthesized cDNA is delivered on ice and stored at $-20^{\circ} \mathrm{C}$.

Real-time quantitative polymerase chain reaction using the Thermal Cycler Dice Real Time System Multiplate RQ (Takara TP800, Tokyo, Japan) and Takara qPCR set, including SYBR Premix ExTaq II, was performed according to the manufacturer's protocol. Four microliter of the first strand cDNA was used in the following assay. The sequences of TFF3 Primers were 5'-AATGCACCTTCTGAGGCACCT-3' (coding sense corresponding to base 452 - 472), 5'-CGTTAAGACATCAGGCTCCAGAT-3' (anti codinf sense corresponding to base 623 - 601) (8). Glyceroldehyde-3-phosphate dehydrogenase (GAPDH) (HA067812, Takara, Tokyo, Japan) and hypoxanthin phosphorobosyltransferase1 (HPRT1) (HA 067805, Takara, Tokyo, Japan) were used as control genes. All primers were purchased from Takara (Tokyo, Japan). 3 steps PCR were used. The conditions for PCR were as follows: $95^{\circ} \mathrm{C}$ for 2 minutes and 50 cycles of $95^{\circ} \mathrm{C}$ for 15 seconds and $60^{\circ} \mathrm{C}$ for 1 minute. Negative control tubes did not contain primers, Calibration tubes contain FTC culture cells cDNA (target sample).

Gel electrophoresis. After amplification of DNA via PCR DNA Gel electrophoresis have been performed to check out quality of amplification of PCR products.

\subsection{Statistical Analysis}

The Mann-Whitney U test was used to compare TFF3mRNA expression level in follicular thyroid adenoma, follicular thyroid carcinoma, adenomatous goiter and normal thyroid tissues using software Multiplate RQ (Takara).

\section{Results}

Study groups included patients, who received surgical treatment according to different surgical diseases of thyroid gland. After conventional histology established final diagnosis formalin-fixed, paraffin-embedded (FFPE) blocks of thyroid tumors were collected for study. The tumor area was checked by HE staining, and only tumor parts were collected from the next continuation sections. Normal tissue, adenomatous goiter, follicular adenoma, minimal invasive follicular carcinoma, wide invasive follicular carcinoma parts were also collected after checking by HE staining (Figure 1).

Patients were assigned into 4 groups: 19 follicular thyroid carcinoma (FTC) cases, 20 follicular thyroid adenoma (FTA) cases, 11 adenomatous goiter (G) cases and 12 samples of normal thyroid tissue $(\mathrm{N})$ adjacent to thyroid tumors were included in the study. There are five cases of FTA were classified as a suspicious follicular thyroid adenoma due to the pathological findings and required follow up. Using the RNeasy FFPE Kit (Qiagen) yielded high concentration of RNA in all cases. Purification and concentration values of extracted total RNA are presented in Table 1.

Table 1. Purification and concentrayion value of total RNA extraxted from FFPE thyroid samples.

FFPE Purification Concentration FFPE Purification Concentration thyroid value (PV): value $(\mathrm{CV})$ : thyroid value $(\mathrm{PV})$ : value $(\mathrm{CV})$ : samples A260/A280 ng/ $\mu \mathrm{l}$ samples A260/A280 $\mathrm{ng} / \mu \mathrm{l}$

\begin{tabular}{|c|c|c|c|c|c|}
\hline \multicolumn{3}{|c|}{$\begin{array}{l}\text { Follicular thyroid } \\
\text { carcinoma (FTC) }\end{array}$} & \multicolumn{3}{|c|}{$\begin{array}{c}\text { Follicular thyroid } \\
\text { adenoma (FTA) }\end{array}$} \\
\hline $\mathrm{C} 1$ & 1.85 & 1532 & A1 & 1.67 & 94 \\
\hline $\mathrm{C} 2$ & 1.75 & 890 & A2 & 1.86 & 1136 \\
\hline $\mathrm{C} 3$ & 1.82 & 1318 & A3 & 1.77 & 542 \\
\hline $\mathrm{C} 4$ & 1.76 & 550 & A4 & 2 & 2212 \\
\hline $\mathrm{C} 5$ & 1.91 & 494 & A5 & 1.9 & 704 \\
\hline C6 & 1.61 & 1466 & A6 & 1.73 & 502 \\
\hline $\mathrm{C} 7$ & 1.81 & 1512 & A7 & 1.8 & 552 \\
\hline $\mathrm{C} 8$ & 1.54 & 278 & A8 & 1.87 & 374 \\
\hline C9 & 1.45 & 122 & A9 & 1.79 & 834 \\
\hline $\mathrm{C} 10$ & 1.88 & 1458 & A 10 & 1.89 & 994 \\
\hline $\mathrm{C} 11$ & 1.61 & 748 & A11 & 1.72 & 234 \\
\hline $\mathrm{C} 12$ & 1.5 & 284 & A12 & 1.5 & 36 \\
\hline $\mathrm{C} 13$ & 1.82 & 1108 & A13 & 1.85 & 100 \\
\hline C14 & 1.84 & 346 & A14 & 1.8 & 622 \\
\hline $\mathrm{C} 15$ & 1.76 & 424 & A15 & 1.81 & 1128 \\
\hline $\mathrm{C} 16$ & 1.79 & 434 & A16 & 2 & 28 \\
\hline $\mathrm{C} 17$ & 2 & 2040 & A17 & 1.79 & 326 \\
\hline $\mathrm{C} 18$ & 1.76 & 1194 & A18 & 1.7 & 674 \\
\hline \multirow[t]{2}{*}{ C19 } & 1.82 & 466 & A19 & 1.78 & 250 \\
\hline & & & A20 & 1.92 & 304 \\
\hline \multicolumn{3}{|c|}{ Adenomatous goiter } & \multicolumn{3}{|c|}{$\begin{array}{l}\text { Normal thyroid tissue } \\
\text { adjacent to thyroid tumors }\end{array}$} \\
\hline G1 & 1.58 & 38 & N1 & 1.73 & 142 \\
\hline G2 & 1.67 & 144 & N2 & 1.52 & 122 \\
\hline G3 & 1.59 & 360 & $\mathrm{~N} 3$ & 1.79 & 312 \\
\hline G4 & 1.79 & 104 & N4 & 1.89 & 496 \\
\hline G5 & 1.48 & 320 & N5 & 1.79 & 520 \\
\hline G6 & 1.31 & 42 & N6 & 1.81 & 80 \\
\hline G7 & 1.5 & 60 & N7 & 1.78 & 232 \\
\hline G8 & 1.79 & 330 & $\mathrm{~N} 8$ & 1.9 & 134 \\
\hline G9 & 1.62 & 130 & N9 & 2 & 48 \\
\hline G10 & 1.14 & 32 & N10 & 2 & 124 \\
\hline G11 & 1.66 & 20 & N11 & 1.58 & 76 \\
\hline \multicolumn{3}{|c|}{ FTC culture cells (target sample) } & N12 & 1.9 & 430 \\
\hline $\mathrm{T} 1$ & 1.78 & 2132 & & & \\
\hline
\end{tabular}




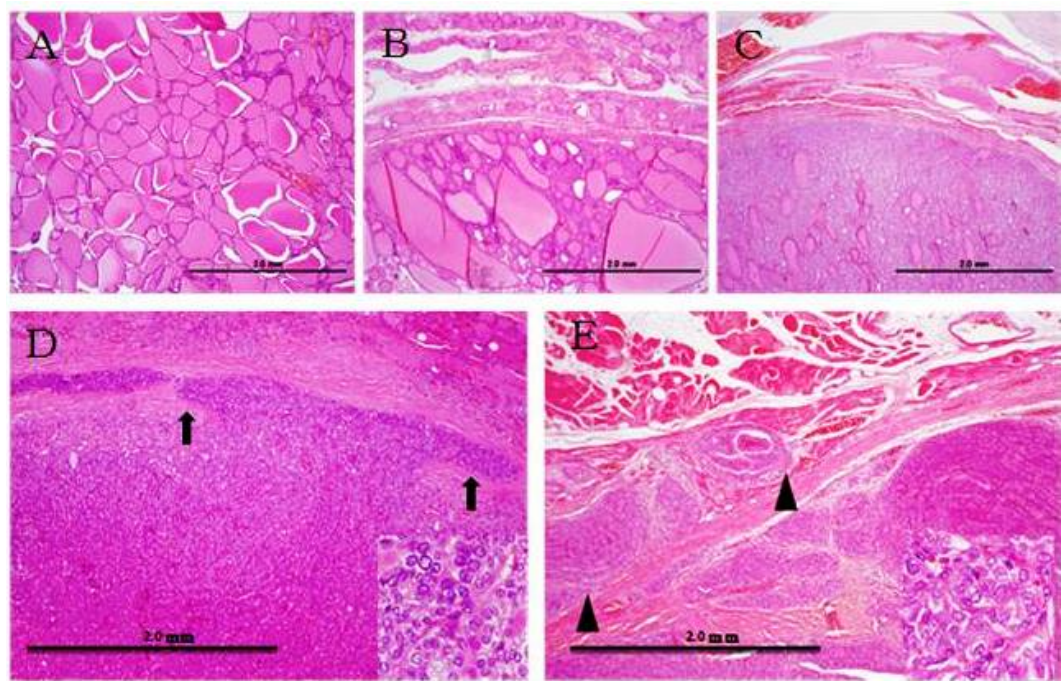

Figure 1. Morphological examination of thyroid tissues. (A) Normal tissue, (B) Adenomatous goiter, (C) Follicular adenoma, (D) Minimal invasive follicular carcinoma, (E) Wide invasive follicular carcinoma. Arrows indicate invasion area. Arrow heads indicate wide invasion $(\times 4)$. Bottom right squares show cell atypia in high magnification $(\times 40)$.

As it is seen from Table 1, all FFPE thyroid samples contain enough amount of RNA for reverse transcription. Concentration values were significantly higher in FTC and FTA relative to $\mathrm{G}$ and $\mathrm{N}$ tissues, possibly due to high density of thyrocytes in the samples. This difference is seen in spectrophotometer curve of Figure 2.

All extracted RNA from samples were immediately reverse transcribed for cDNA synthesis.

In order to check the conditions of the real time PCR, gel electrophoresis was performed for the sample of each group. The amplified band was detected an expected position in all samples. Real time PCR was performed on this condition (Figure 3).

Synthesized cDNA is used in real-time PCR for relative quantification of TFF3 mRNA expression level in all samples. When the appropriate cutoff point was set and questionable cases related to contamination and not amplified samples during the RT-qPCR were excluded we get results as in Figure 4.

Relative quantification of TFF3 mRNA expression level showed broad ranges both in FTC and FTA (2554.8 \pm 1321.9 and $5244.3 \pm 3796.2$ respectively, $\mathrm{P}>0.05$ ), while the analyses in $\mathrm{G}$ and $\mathrm{N}$ tissues indicated narrow ranges $(2492 \pm 601.7$ and $4939.7 \pm 440.9$ respectively, $\mathrm{P}$ $<0.05$ ). Our findings showed broad range of TFF3 mRNA expression level both in FTC and FTA, while the analyses in $\mathrm{G}$ and $\mathrm{N}$ tissues indicated narrow ranges using descriptive statistics (Figure 4).

In our study TFF3 does not perfectly match with the pathological diagnosis of follicular thyroid tumors, though we can see tendency of upregulation and downregulation of TFF3 mRNA expression level in different thyroid tumors while grading the results. Downregulation of TFF3

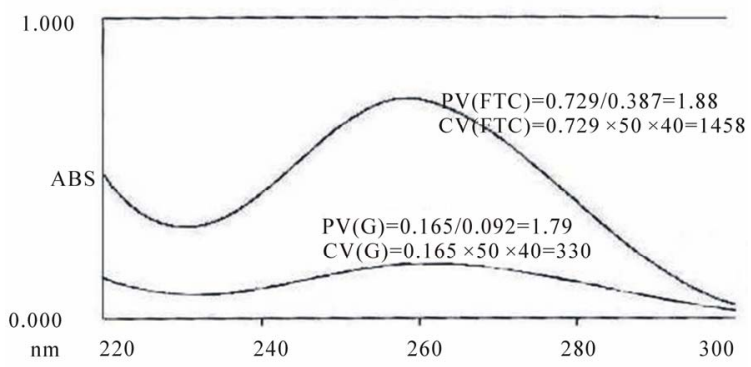

Figure 2. Spectrophotometer curve of RNA yield of FTC and G FFPE tissue.
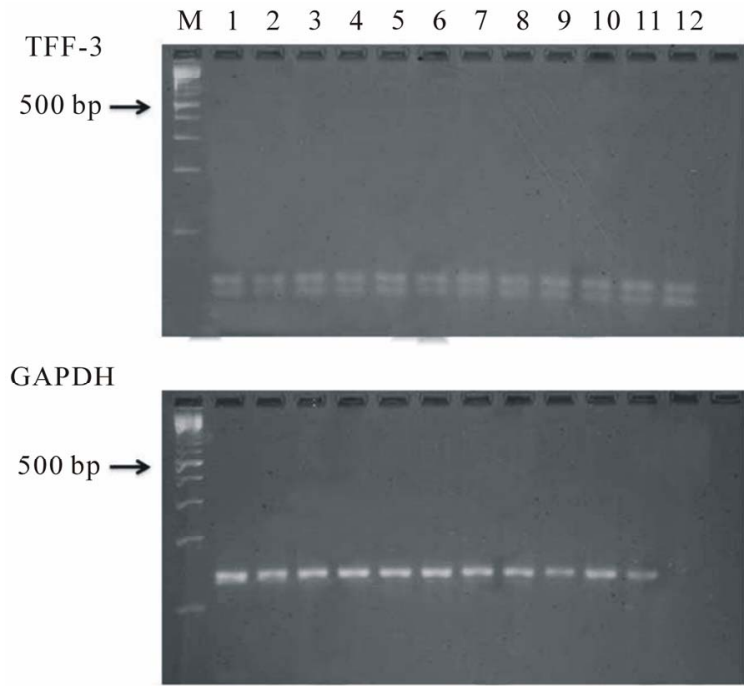

Figure 3. Gel electrophoresis analyses of RT-PCR products of TFF3 and GAPDH M: 100 bp radar size marker, 1 - 3 FTC, 4 - 6 FTA, 7 - 9 adenomatous goiter, 10 - 12 normal thyroid tissue. 


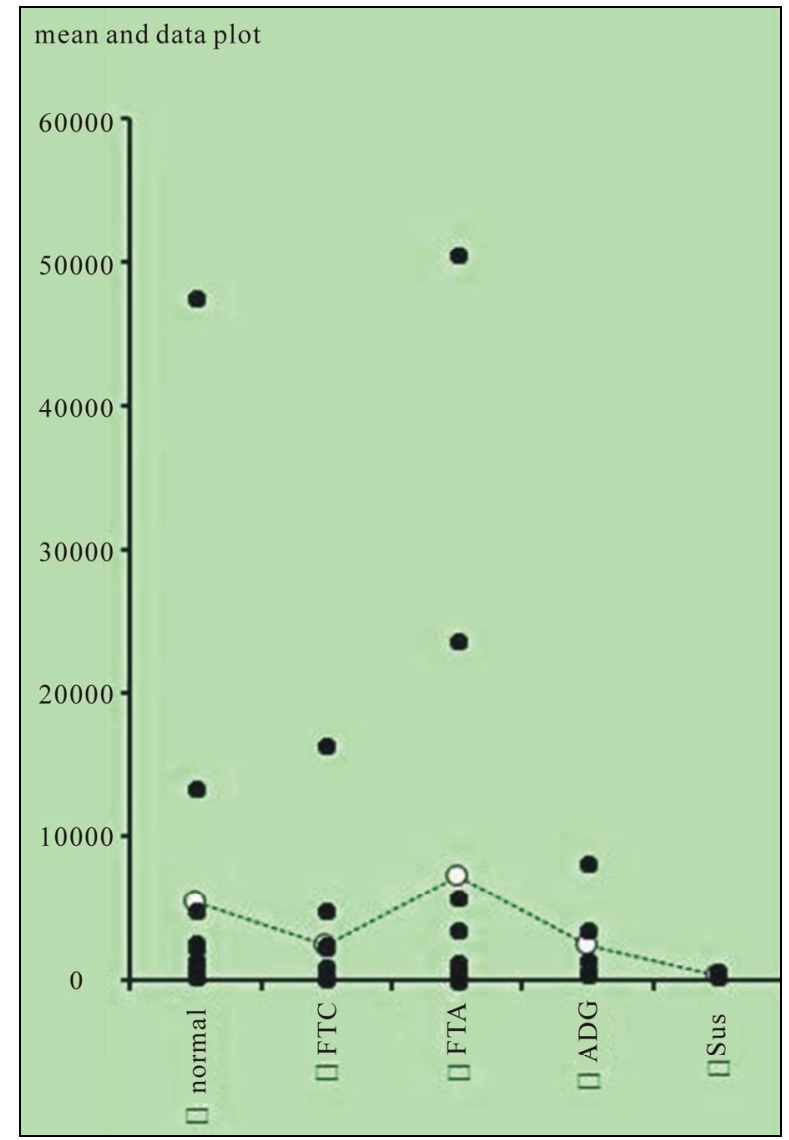

Figure 4. Results of relative quantification of TFF3 mRNA expression level using RT-qPCR. FTC, follicular thyroid carcinoma; FTA, follicular thyroid adenoma; ADG, adenomatous goiter; Sus, suspicious follicular thyroid adenoma that require follow up as pathologist.

is observed in $67 \%$ FTC and in $100 \%$ suspicious follicular thyroid adenoma that require follow up as pathologist indicated, while upregulation of TFF3 is observed in $53 \%$ FTA. TFF3 levels are evenly distributed in normal thyroid tissues and adenomatous goiters.

\section{Discussion}

Differential expression of TFF3 mRNA between follicular adenomas and carcinomas was first reported in detail by Takano T. et al., using serial analysis of gene expression (SAGE) in 2004 [7]. Authors estimated the efficiency of differential diagnosis between follicular adenoma and carcinoma by measuring the expression levels of TFF3 mRNA. In the 54 follicular adenomas and 29 follicular carcinomas analyzed, the relative expression of TFF3 mRNA was markedly decreased in 7 follicular carcinomas of the widely invasive type and with evident distant metastases. When the appropriate cutoff point was set and pathologically questionable cases were excluded, the sensitivity, specificity and accuracy were
$80.0 \%, 91.5 \%$, and $87.5 \%$, respectively [6].

Taniguchi et al. used a unique system for analysis of gene expression called Adapter-Tagged competitive PCR, to screen over 2000 genes expressed in thyroid follicular tumors and concluded that TFF3 mRNA is one of the most promising markers for diagnosing follicular carcinoma [12]. Foukakis et al. reevaluated 26 previously reported candidate genes and found that the combination of TFF3 and hTERT mRNA could most efficiently distinguish follicular carcinomas from adenomas [11]. Another re-evaluation had performed by Krause et al., who examined 10 candidate genes and found that downergulation of TFF3 in malignant tumors was the best indicator for thyroid malignancies including follicular carcinoma and any combination assays that include TFF 3 could efficiently distinguish follicular carcinomas from adenomas [13].

Molecular-based diagnosis of anaplastic and papillary carcinomas can be established easily with genes overexpressed restrictedly in these tumors in clear contrast to follicular carcinoma [17-19]. The reason why TFF3 is downregulated in follicular carcinomas is not clear at present. It is also unclear how far environmental factors, such as iodine supply, may influence the candidate gene expression. The further investigation to clarify this phenomenon may give a clue to understand the very fundamental mechanism of thyroid carcinogenesis.

Differential diagnosis FTC from FTA is often difficult since presence or absence of capsular/vascular invasion can not be determined by preoperative fine needle aspiration cytology, and may not be judged unanimously on permanent sections even among experienced pathologists. Determination of molecular-genetic factors such as TFF3 mRNA in the follicular thyroid tumors may be useful aid to improve the accuracy of diagnosis. In fact, our results showed that expression level of TFF3 mRNA was associated with histopathological diagnoses, although the relationship should be interpreted with caution. Though we found that $100 \%$ suspicious follicular thyroid adenomas and $67 \%$ of FTC correlate with downregulation of TTF3. We speculate that some other risk factors are also should be taken into account while determining subgroup of patients with TFF3 downregulation and upregulation. Perhaps TFF3 must be analyzed in correlation with other risk factors such as TNM, age, gender, $\mathrm{Tg}$ and others to identified subgroups with significant data of TFF3 upregulation and downregulation appropriate to these correlations.

Another important point is that we tried to confirm the utility of this approach by using archival thyroid tissuesFFPE blocks. Human surgical tissue samples are routinely fixed with formalin and then embedded in paraffin for long-term preservation. The extensive histopathology 
archive of FFPE tissue samples represents an extremely valuable resource for retrospective studies of gene expression patterns in diseased tissues. Limitations on using these samples for mRNA expression studies have been imposed by the significant modification and degradation of RNA during tissue fixation, resulting in poor yield of RNA. In our study we revealed that FFPE blocks of thyroid diseased tissue with the archival time 1 - 5 years can be used for RNA extraction with the high yield of RNA. Purification values were in average 1.8. Concentration values were significantly higher in FTC and FTA relative to $\mathrm{G}$ and $\mathrm{N}$ tissues, possibly due to high density of thyrocytes in the samples.

Determination of molecular-genetic factors such as TFF3 mRNA in correlation with other risk factors in the follicular thyroid tumors may be useful aid to improve the accuracy of diagnosis, though it is considered to be unstable and relatively low concentrated genetic substance. Results of our study indicate the applicability of mRNA expression patterns as potential markers of human cancer, and our results suggest that FFPE tissues are suitable resources for mRNA expression analyses. FFPE tissues from thyroid follicular tumors can be used for measurement of unstable and low concentrated genetic substances such as TFF3 mRNA. Meanwhile, its diagnostic value yet remains to be determined.

\section{Acknowledgements}

We wish to thank the patients whose samples were used for this research. We also thank all Pathology Department and Endocrine Surgery Department stuff of the TWMU for their substantial help, supplying materials, kits and technical assistance during carrying out this research. We thank Dr. Toru Takano, from Department of Laboratory Medicine, Osaka University Graduate School of Medicine, Japan for his advises in the planning of this research. This research was performed with support of the fellowship program of Matsumae International Foundation, Tokyo, Japan.

\section{REFERENCES}

[1] M. Eszlinger, K. Krohn, S. Hauptmann, H. Dralle, T. Giordano and R. Paschke, "Perspectives for Improved and More Accurate Classification of Thyroid Epithelial Tumors," Journal of Clinical Endocrinology \& Metabolism, Vol. 93, No. 9, 2008, pp. 3286-3294.

[2] M. Pulcrano, H. Boukheris, M. Talbot, B. Caillou, C. Dupuy, A. Virion, V. F. De and M. Schlumberger, "Poorly Differentiated Follicular thyroid carcinoma: Prognostic Factors and Relevance of Histological Classification," Thyroid, Vol. 17, No. 7, 2007, pp. 639-646 doi:10.1089/thy.2007.0029

[3] B. Hamberger, H. Gharib, L. J. Melton 3rd, J. R. Goellner and A. R. Zinsmeister, "Fine-Needle Aspiration Biopsy of Thyroid Nodules. Impact on Thyroid Practice and Cost of Care," American Journal of Medicine, Vol. 73, No. 3, 1982, pp. 381-384. doi:10.1016/0002-9343(82)90731-8

[4] J. M. Cerutti, R. Delcelo, M. J. Amadei, C. Nakabashi, R. M. Maciel, B. Peterson, J. Shoemaker and G. J. Riggins, "Apreoperative Diagnostic Test That Distinguishes Benign from Malignant Thyroid Carcinoma Based on Gene Expression," Journal of Clinical Investigation, Vol. 113, No. 8, 2004, pp. 1234-1242.

[5] F. Weber, L. Shen, M. A. Aldred, C. D. Morrison, A. Frilling, M. Saji, F Schuppert, C. E. Broelsch, M. D. Ringel and C. Eng, "Genetic Classification of Benign and Malignant Thyroid Follicular neoplasia Based on a ThreeGene Combination," Journal of Clinical Endocrinology \& Metabolism, Vol. 90, No. 5, 2005, pp. 2512-2521. doi:10.1210/jc.2004-2028

[6] T. Takano, A. Miyauchi, H. Yoshida, K. Kuma and N. Amino, "Decreased Relative Expression Level of Trefoil Factor 3 mRNA to Galectin-3 mRNA Distinguishes Thyroid Follicular carcinoma from Adenoma," Cancer Letters, Vol. 219, No. 1, 2005, pp. 91-96.

doi:10.1016/j.canlet.2004.07.004

[7] T. Takano, A. Miyauchi, H. Yoshida, K. Kuma and N. Amino, "High-Throughput Differential Screening of mRNAs by Serial Analysis of Gene Expression: Decreased Expression of Trefoil Factor 3 mRNA in Thyroid Follicular carcinomas," British Journal of Cancer, Vol. 90, No. 8, 2004, pp. 1600-1605. doi:10.1038/sj.bjc.6601702

[8] T. Takano and H. Yamada, "Trefoil Factor 3 (TFF3): A Promising Indicator for Diagnosing Thyroid Follicular Carcinoma. Review," Endocrine Journal, Vol. 56, No. 1, 2009, pp. 9-16.

[9] M. Eszlinger, K. Krohn, K. Berger, J. Lauter, S. Kropf, M. Beck, D. Fuhrer and R. Paschke, "Gene Expression Analysis Reveals Evidence for Increased Expression of Cell Cycle-Associated Genes and Gq-Protein-Protein Kinase C Signaling in Cold Thyroid Nodules," Journal of Clinical Endocrinology \& Metabolism, Vol. 90, No. 2, 2005, pp. 1163-1170. doi:10.1210/jc.2004-1242

[10] D. Fuhrer, M. Eszlinger, S. Karger, K. Krause, C. Engelhardt, D. Hasenclever, H. Dralle and R. Paschke, "Evaluation of Insulin-Like Growth Factor II, Cyclooxygenase2, Ets-1 and Thyroid-Specific Thyroglobulin mRNA Expression in Benign and Malignant Thyroid Tumours," European Journal of Endocrinology, Vol. 152, No. 5, 2005, pp. 785-790. doi:10.1530/eje.1.01912

[11] T. Foukakis, A. Gusnanto, A. Y. Au, A. Hoog, W. O. Lui, C. Larsson, G. Wallin and J. Zedenius, "A PCR-Based Expression Signature of Malignancy in Follicular Thyroid Tumors," Endocrine-Related Cancer, Vol. 14, No. 2, 2007, pp. 381-391. doi:10.1677/ERC-06-0023

[12] K. Taniguchi, T. Takano, A. Miyauchi, K. Koizumi, Y. Ito, Y. Takamura, M. Ishitobi, Y. Miyoshi, T. Taguchi, Y. Tamaki, K. Kato and S. Noguchi, "Differentiation of Follicular Thyroid Adenoma from Carcinoma by Means of Gene Expression Profiling with Adapter-Tagged Competitive Polymerase Chain Reaction," Oncology, Vol. 69, No. 5, 2005, pp. 428-435. doi:10.1159/000089998 
[13] K. Krause, M. Eszlinger, O. Gimm, S. Karger, C. Engelhardt, H. Dralle and D. Fuhrer, "TFF3 Based Candidate Gene Discrimination of Benign and Malignant Thyroid Tumours in a Region with Borderline Iodine Deficiency," Journal of Clinical Endocrinology \& Metabolism, Vol. 93, No. 4, 2008, pp. 1390-1393.

[14] L. Jin, et al., "HMGA2 Expression Analysis in Cytological and Paraffin-Embedded Tissue Specimens of Thyroid Tumors by Relative Quantitative RT-PCR," Diagnostic Molecular Pathology, Vol. 20, No. 2, 2011, pp. 71-80. doi:10.1097/PDM.0b013e3181ed784d

[15] M. T. Tetzlaff, et al., "Differential Expression of MiRNAs in Papillary Thyroid Carcinoma Compared to Multinodular Goiter Using Formalin Fixed Paraffin Embedded Tissues," Endocrine Pathology, Vol. 18, No. 3, 2007, pp. 163-173. doi:10.1007/s12022-007-0023-7

[16] T. Takano, F. Matsuzuka, H. Sumizaki, K. Kuma and N. Amino, "Rapid Detection of Specific Messenger RNAs in Thyroid Carcinomas by Reverse Transcription-PCR with
Degenerate Primers: Specific Expression of Oncofetalfibronectin Messenger RNA in Papillary Carcinoma," Cancer Research, Vol. 57, No. 17, 1997, pp. 3792-3797.

[17] T. Takano, A. Miyauchi, T. Yokozawa, F. Matsuzuka, G. Liu, T. Higashiyama, S. Morita, K. Kuma and N. Amino, "Accurate and Objective Preoperative Diagnosis of Thyroid Papillary Carcinomas by Reverse Transcription-PCR Detection of Oncofetalfibronectin Messenger RNA in Fine-Needle Aspiration Biopsies," Cancer Research, Vol. 58, No. 21, 1998, pp. 4913-4917.

[18] T. Takano, A. Miyauchi, T. Yokozawa, F. Matsuzuka, I. Maeda, K. Kuma and N. Amino, "Preoperative Diagnosis of Thyroid Papillary and Anaplastic Carcinomas by RealTime Quantitative Reverse Transcription-Polymerase Chain Reaction of Oncofetalfibronectin Messenger RNA," Cancer Research, Vol. 59, No. 18, 1999, pp. 4542-4545.

[19] S. Ismailov, M. Rashitov and L. Nugmanova, "Thyroid Tumors," Methodical Handbook, Tashkent, 2007, p. 132. 